\title{
Microalbuminuria in patients with rheumatoid arthritis
}

\author{
Lars Møller Pedersen, Henrik Nordin, Birthe Svensson, Henning Bliddal
}

\begin{abstract}
Objectives-To assess (a) the prevalence of microalbuminuria in patients with rheumatoid arthritis, (b) the association between urinary albumin excretion and disease activity as estimated by the erythrocyte sedimentation rate and $C$ reactive protein (CRP), and (c) the association between urinary albumin excretion and treatment with antirheumatic drugs.
\end{abstract}

Methods-Sixty five patients with rheumatoid arthritis attending two rheumatology clinics were compared with 51 control subjects matched by age and sex. The controls consisted of 20 healthy subjects, 16 patients with osteoarthritis and 15 with non-articular rheumatism. Patients with hypertension, diabetes mellitus, or evidence of previous renal disease were not included. Urinary albumin was assayed by immunoturbidimetry in random urine samples on two occasions within seven months. The results were expressed as the ratio of urinary albumin to urinary creatinine ratio. Disease activity was assessed by the erythrocyte sedimentation rate and CRP. A drug history for the year before entry to the study was obtained for each patient.

Results-Urinary albumin to creatinine ratio in patients with rheumatoid arthritis was significantly greater than in controls $(p<0.01)$. Microalbuminuria (urinary albumin to creatinine ratio $3-30 \mathrm{mg} / \mathbf{m m o l}$ in either or both urine samples) was present in $27 \cdot 7 \%$ of patients with rheumatoid arthritis and $7.8 \%$ of the control subjects. A significant relation was noted between urinary albumin to creatinine ratio and CRP, and the duration of disease. The number of patients treated with either gold or penicillamine was significantly greater in patients with microalbuminuria than in patients with normoalbuminuria.

Conclusions-Microalbuminuria is frequently present in patients with rheumatoid arthritis. Treatment with gold and penicillamine seems to increase the risk of developing microalbuminuria. Urinary albumin measured by immunochemical methods is a simple and sensitive test to detect early subclinical renal dysfunction and drug induced renal damage in rheumatoid arthritis. Urinary albumin excretion was found to be significantly correlated with CRP and may be a sensitive indicator of disease activity in patients with rheumatoid arthritis.

(Ann Rheum Dis 1995; 54: 189-192)

Microalbuminuria has been defined as an increased excretion of albumin above the reference range for healthy subjects which is undetectable by dipstick testing. ${ }^{1}$ It is associated with a generalised vascular damage in patients with diabetes mellitus and is a predictor of diabetic nephropathy. ${ }^{2}{ }^{3}$ In nondiabetic populations, microalbuminuria has been found with a prevalence ranging from $6.3 \%$ to $13 \%{ }^{4}$ Further longitudinal studies are required to elucidate the long term renal prognosis in non-diabetic subjects with the condition. However, increased urinary albumin excretion is associated with hypertension, cardiovascular disease, and increased mortality rate, ${ }^{5-7}$ and has also been reported in patients with rheumatic diseases other than rheumatoid arthritis (RA), such as systemic lupus erythematosus ${ }^{89}$ and systemic sclerosis. ${ }^{10}$

In patients with RA there is a high prevalence of renal impairment, with evidence of reduced glomerular filtration and tubular function. ${ }^{11-13}$ Various morphological findings in the kidneys have been described ${ }^{111415}$ and renal disease is presumed to be a frequent cause of death in RA. ${ }^{16}{ }^{17}$ It would be useful, therefore, to identify those at risk of developing clinical nephropathy, and sensitive measures of renal function should be available. In RA, glomerular proteinuria has been considered as a complication of advanced disease caused by the direct effects of the disease on the kidney, or the action of nephrotoxic drugs, or both. However, subclinical renal dysfunction is not uncommon in RA, and many of these patients with incipient nephropathy are not detected by routine laboratory tests such as assays for urine total protein or Albustix. ${ }^{18} 19$ The advent of assays sensitive and specific for urinary albumin has enabled the detection of glomerular abnormalities at an earlier stage in patients without clinical renal involvement. ${ }^{20}$ Patients at risk of developing renal dysfunction might therefore be detected earlier by the appearance of microalbuminuria. Furthermore, increased urinary excretion of albumin may reflect not only glomerular disease, but also the inflammatory state and disease activity. The aim of the present study was to evaluate the frequency of microalbuminuria in 
patients with RA in order to establish a reliable method for an accurate assessment of subclinical renal dysfunction.

\section{Patients and methods}

Sixty five patients (44 female) aged 23-82 years (mean 63.4 years) with $R A$, and 51 controls attending two specialised outpatient clinics of rheumatology were examined. All RA patients fulfilled the ARA 1987 revised criteria. ${ }^{21}$ They were selected by the criteria of serum creatinine in the normal range (less than $120 \mu \mathrm{mol} / \mathrm{l}$ ) and no evidence of previous renal disease. Patients with known past or present hypertension, congestive heart failure, or diabetes mellitus were not included. The controls also met the criteria and were matched by age and gender (table 1 ). They comprised $20(39 \cdot 2 \%)$ healthy subjects, $16(31 \cdot 4 \%)$ patients with osteoarthritis, and $15(29.4 \%)$ with non-articular rheumatism.

Random urine samples were collected from all patients during routine assessment on two occasions within seven months (mean $3 \cdot 2$ months). Urine specimens were collected in the morning, but not at a fixed time. On the same occasions blood was taken and disease activity assessed by measuring erythrocyte sedimentation rate (ESR) (Westergren) and $C$ reactive protein (CRP). A drug history was obtained from each patient. All nonsteroidal anti-inflammatory drugs (NSAIDs) and disease modifying antirheumatic drugs (DMARDs) prescribed during the year before entry to the study were noted.

Urinary albumin was assayed by immunoturbidimetry using rabbit anti-human albumin (Dakopatts $\mathrm{A} / \mathrm{S}, \mathrm{DK}$ ) and a Cobas Mira discretionary analyser (F HoffmanLa Roche \& Co. Ltd, Diagnostica, Basle, Switzerland). ${ }^{22}$ Urinary creatinine was measured by an end point Jaffé reaction. For standardisation of urine concentration, urinary albumin was expressed as the ratio of urinary albumin to urinary creatinine $(\mathrm{mg} / \mathrm{mmol})$. Urine samples positive to Labstix (Ames) for glucose, protein, blood, leucocytes, or nitrites were rejected.

The variables were calculated as the average of the two samples collected in each patient. Since the data studied were not normally distributed, non-parametric methods were applied. For unpaired data, analyses were performed with the Mann-Whitney test. Correlations were evaluated using Spearman's rank correlation coefficient $(r)$. Subjects were classified as having microalbuminuria if they had an albumin:creatinine ratio of

Table 1 Characteristics of $R A$ and control subjects

\begin{tabular}{lcc}
\hline & $R A$ & Controls \\
\hline No of patients & 65 & 51 \\
Female/male ratio & $2 \cdot 3$ & $2 \cdot 2$ \\
Age (years) & $63 \cdot 4(11 \cdot 3)$ & $61 \cdot 8(11 \cdot 7)$ \\
Blood pressure (mm Hg) & $138(13)$ & $131(10)$ \\
$\quad$ Systolic & $78(6)$ & $75(5)$ \\
$\quad$ Diastolic & $94(19)$ & $84(16)$ \\
Serum creatinine $(\mu \mathrm{mol} / \mathrm{l})$ &
\end{tabular}

Mean values (SD).
Table 2 Prevalence of microalbuminuria in patients with $R A$ and controls

\begin{tabular}{lll}
\hline Group & \multicolumn{2}{l}{ Microalbuminuria present } \\
\cline { 2 - 3 } & One or both samples & Neither sample \\
\hline RA $(n=65)$ & $18(27 \cdot 7 \%)$ & $47(72 \cdot 3 \%)$ \\
Controls $(n=51)$ & $4(7 \cdot 8 \%)$ & $47(92 \cdot 2 \%)$ \\
\hline
\end{tabular}

$3-30 \mathrm{mg} / \mathrm{mmol}$ in either or both of the urine samples. ${ }^{23} \chi^{2}$ was used for the comparison of proportions. $p$ less than 0.05 was considered significant. Ethical approval was obtained from the Copenhagen Research Ethics Committee, and patients gave their informed consent before entering the study.

\section{Results}

The prevalence of microalbuminuria (albumin:creatinine ratio $3-30 \mathrm{mg} / \mathrm{mmol}$ in either or both urine samples) differed significantly between patients with RA and controls $(27.7 \% \vee 7.8 \% ; \mathrm{p}<0.01)$ (table 2$)$. None of the urine samples exceeded the upper limit of the range defined as microalbuminuria. The median value of albumin:creatinine ratio in RA patients was significantly greater than that in controls $(2.83 v 2.28 ; \mathrm{p}<0.01,95 \%$ confidence limits of median difference 0.26 to 1.54). There was no significant difference in albumin:creatinine ratio between the three control subgroups $(2 \cdot 16 v 2.47 v 2 \cdot 27 ; \mathrm{p}<0 \cdot 1)$, but that between the two urine samples was significantly correlated $(p<0.001)$; the median ratio did not differ between men and women in RA patients $(\mathrm{p}<0 \cdot 1)$ and control groups $(p<0 \cdot 2)$. The albumin:creatinine ratio was not significantly associated with age $(p<0 \cdot 1)$. Patients with microalbuminuria had a significantly greater median duration of RA than the group with normal albumin:creatinine ratio $(11.2 v 7.8$ years; $p<0.001)$.

Median values (ranges) for the measures of rheumatoid disease activity were 112 (16-1615) nmol/1 for CRP and 19 (3-88) $\mathrm{mm} /$ 1 st $\mathrm{h}$ for ESR. CRP was significantly correlated with urinary albumin:creatinine ratio (figure), but no significant relation was found between ESR and the ratio (figure). The majority of the RA patients was exposed to both NSAIDs and DMARDs (table 3). The frequency of treatment with gold and penicillamine was significantly greater in patients with microalbuminuria than in patients with normal urinary excretion of albumin. Sixty one percent of patients with microalbuminuria were treated with either gold or penicillamine. Microalbuminuria was demonstrated in $58 \%$ of patients exposed to gold or penicillamine and

Table 3 Urinary albumin excretion in $R A$ patients related to drug therapy in the year before time of study

\begin{tabular}{|c|c|c|c|}
\hline & $\begin{array}{l}\text { Normoalbuminuria } \\
\text { (\%) }(n=47)\end{array}$ & $\begin{array}{l}\text { Microalbuminuria } \\
\text { (\%) }(n=18)\end{array}$ & $p$ \\
\hline $\begin{array}{l}\text { NSAIDs } \\
\text { Gold } \\
\text { Penicillamine } \\
\text { Methotrexate } \\
\text { Sulphasalazine } \\
\text { Hydroxychloroquine } \\
\text { Azathioprine }\end{array}$ & $\begin{array}{r}76 \cdot 6 \\
8 \cdot 5 \\
8 \cdot 5 \\
12 \cdot 8 \\
17 \cdot 0 \\
8 \cdot 5 \\
2 \cdot 1\end{array}$ & $\begin{array}{l}77 \cdot 8 \\
33 \cdot 3 \\
27 \cdot 8 \\
11 \cdot 1 \\
11 \cdot 1 \\
5 \cdot 6 \\
0\end{array}$ & $\begin{array}{l}\text { NS } \\
0 \cdot 01 \\
0 \cdot 04 \\
\text { NS } \\
\text { NS } \\
\text { NS } \\
\text { NS }\end{array}$ \\
\hline
\end{tabular}



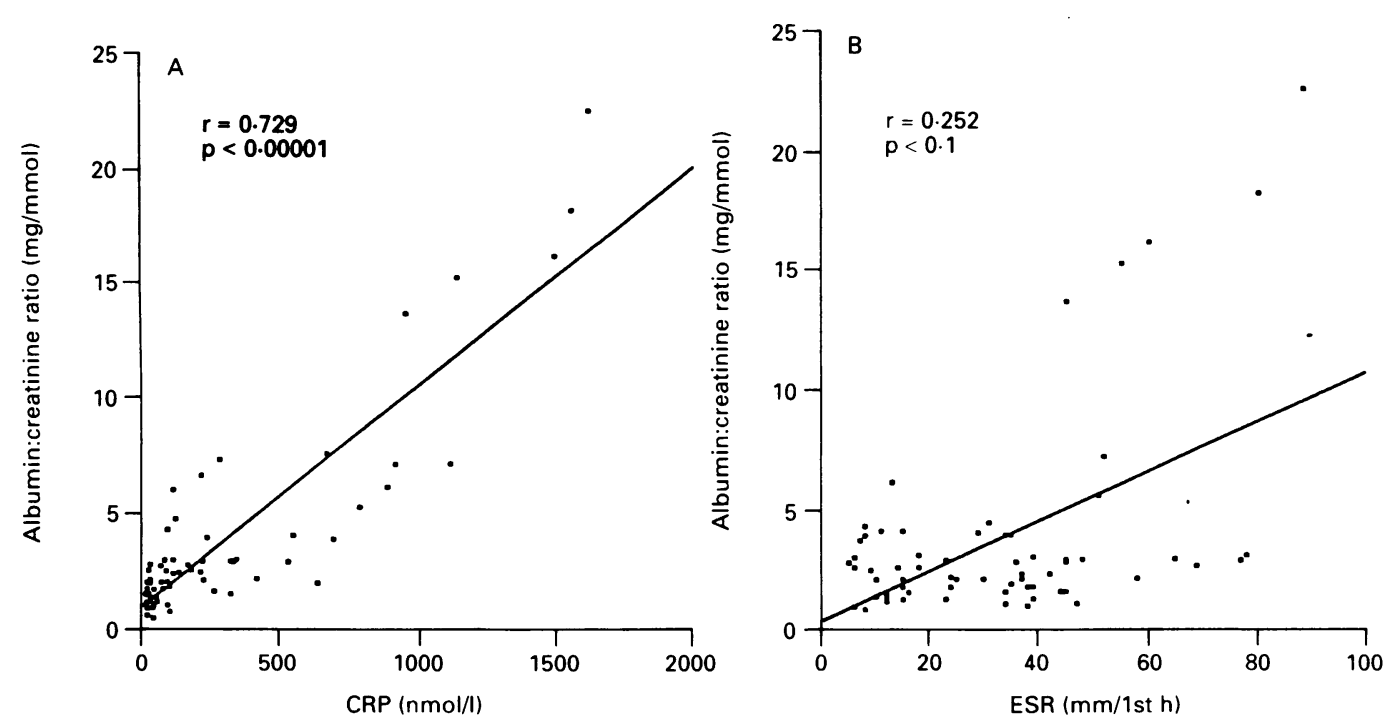

Figure 1 Correlations between albumin:creatinine ratio and markers of disease activity estimated by $C$ reactive protein $(C R P)(A)$ and erythrocyte sedimentation rate $(E S R)(B)$ in $R A$ patients $(n=65)$.

$21 \%$ of patients treated with other DMARDs. There was no difference in treatment with NSAIDs between patients with normal urinary albumin and those with microalbuminuria.

\section{Discussion}

Patients with RA are at risk of developing renal complication $\mathrm{s}^{24}$ and proteinuria increases the mortality rate. ${ }^{26}$ It is therefore of crucial importance to have a sensitive method for reliable measurement of renal dysfunction in clinical practice. Our report confirms the presence of pathological albuminuria in many RA patients without a history of renal dysfunction, hypertension, or diabetes mellitus. Our findings are consistent with earlier reports concerning subclinical renal dysfunction in RA. ${ }^{12} 27$

Renal involvement may remain unnoticed for a long period in a reversible subclinical stage and should be detected as early as possible. ${ }^{25}$ Routine measures for renal function such as assays for urine total protein, urine dipstick testing, urine cytology, and serum creatinine may not reflect the renal changes until severe renal damage has occurred. In our study, all patients with microalbuminuria had a normal serum creatinine concentration and gave a negative result with Albustix. Because of the availability of sensitive and specific immunological assays for urine albumin, it is now possible to detect glomerular dysfunction at an early stage. Urinary albumin measured by immunoturbidimetry or other immunochemical methods is a simple and sensitive test of early subclinical renal damage. ${ }^{20}$

Some studies have favoured the possibility of a renal lesion specific to rheumatoid disease itself, ${ }^{12} 28{ }^{29}$ but it seems most likely that the combined influence of drugs, RA itself, and other conditions unrelated to RA (for example diabetes mellitus, hypertension, infection) are responsible for most cases of severe renal dysfunction. The majority of antirheumatic drugs have been documented to affect glomerular and tubular function. ${ }^{30} 31$ Renal function impairment resulting from treatment with antirheumatic drugs may be underestimated, as it is often asymptomatic. ${ }^{31}$ If adverse renal effects are to be reduced to a minimum, sensitive tests of renal function should be available. Testing for microalbuminuria is a simple screening procedure in patients treated with nephrotoxic drugs. The method could be used to detect early renal dysfunction and monitor patients at risk, especially those who require therapy with drugs which are nephrotoxic, providing an accurate clinical assessment of glomerular function and a rational therapeutic approach.

In the present study, most patients were treated with NSAIDs, often combined with DMARDs. Treatment with penicillamine and gold was associated significantly with microalbuminuria. These findings are consistent with other studies suggesting that these drugs are important contributors to proteinuria and microalbuminuria in patients with RA. ${ }^{32}$ Because of the complexity of rheumatoid drug histories, it is difficult to correlate results with individual drug treatments and only a few of the patients in our study did not receive antirheumatic drugs. We cannot, therefore, conclude from our results if microalbuminuria is solely a drug induced side effect.

It has been suggested that changes in renal permeability to plasma proteins reflect increased systemic vascular permeability in acute inflammatory conditions. ${ }^{33}$ Thus the urinary excretion of albumin may reflect a systemic reaction in the acute phase response. ${ }^{34}$ In our study a highly significant correlation between CRP and urinary excretion of albumin was demonstrated. However, the correlation with ESR was not significant. The latter is partially explained by some patients with normoalbuminuria who had increased values of ESR for reasons other than RA; it may also reflect a low sensitivity of ESR as a marker of disease activity. Microalbuminuria was associated with the duration of disease. The 
possible correlation between urinary excretion of albumin, disease activity, and duration of RA may be explained in two ways: either severe and long standing RA tends to affect the kidneys and the systemic vascular permeability more, or patients with more severe and long standing disease receive more nephrotoxic treatment. Urine analyses reflecting renal tubular function have been shown to correlate with disease activity in RA. ${ }^{12}$ We have found an association between urinary excretion of albumin and CRP, but further investigations are required using more sensitive indicators of disease activity.

In conclusion, microalbuminuria and subclinical renal damage are frequent in RA, particularly in those with long standing disease. A subclinical renal involvement may not be revealed by routine laboratory tests such as serum creatinine. Our results suggest that microalbuminuria is a more sensitive predictor of renal dysfunction in patients at risk. Its measurement may serve as a useful tool for the management of patients with RA but without clinical nephropathy. However, the long term renal prognosis in patients with microalbuminuria requires clarification in longitudinal studies. We believe that in the majority of patients with microalbuminuria in RA, the problem is reversible and rarely develops to end stage renal failure. The effects of many antirheumatic drugs on glomerular and tubular function can be of clinical importance and should be monitored with sensitive methods. We recommend immunological methods measuring urinary excretion of albumin as a routine procedure to detect glomerular involvement in its initial phase in order to devise the most appropriate treatment in patients with RA. Further studies are recommended to clarify a possible association between urinary excretion of albumin and disease activity, and to evaluate microalbuminuria as an indicator of the long term prognosis in RA.

1 Viberti G C, Wiseman M J. The kidney in diabetes: significance of the early abnormalities. Clin Endocrino Metab 1986; 15: 783-806.

2 Viberti G C, Hill R D, Jarret R J, Argyropoulos A, Mahmud U, Keen H. Microalbuminuria as a predictor of clinical nephropathy in insulin-dependent diabetes clinical nephropathy in insul
mellitus. Lancet 1982; i: 1430-2.

3 Deckert T, Feldt-Rasmussen B, Borch-Johnsen K, Jensen T, Kofoed-Enevoldsen A. Albuminuria reflects widespread vascular damage. The Steno hypothesis. Diabetologia 1989; 32: 219-26.

4 Winocour P H, Harland J O E, Millar J P, Laker M F, Alberti K G M M. Microalbuminuria and associated cardiovascular risk factors in the community. Atherosclerosis 1992; 93: 71-81.

5 Parving H H, Jensen H A, Mogensen C E, Evrin P E. Increased urinary albumin excretion rate in benign essential hypertension. Lancet 1974; i: 1190-2.
6 Yudkin J S, Forrest R D, Jackson C A. Microalbuminuria as predictor of vascular disease in non-diabetic subjects. Lancet 1988; ii: $530-3$.

7 Damsgaard E M, Frøland A, Jørgensen O D, Mogensen C E. Microalbuminuria as predictor of increased mortality in Microalbuminuria as predictor of increased

8 Yamada A, Miyakawa Y, Shibata S, Kosaka K. Radioimmunoassay of urine albumin in subclinical lupus nephritis. N Engl f Med 1980; 11: 643.

9 Terai C, Nojima Y, Takano K, Yamada A, Takaku F. Determination of urinary albumin excretion by radioimmunoassay in patients with subclinical lupus nephritis. Clin Nephrol 1987; 27: 79-83.

10 Dawnay A, Wilson A G Mc T, Lamb E, Kirby J D T, Cattell W R. Microalbuminuria in systemic sclerosis. Ann Rheum Dis 1992; 51: 384-8.

11 Boers M, Croonen A M, Dijkmans A C, et al. Renal findings in rheumatoid arthritis: clinical aspects of 132 necropsies. Ann Rheum Dis 1987; 46: 658-63.

12 Dieppe P A, Doyle D V, Burry H C, Tucker S M. Renal disease in rheumatoid arthritis. BMf 1976; $1: 611-2$.

13 Hordon L D, Bird H A, Cooper E H. Renal tubular dysfunction in rheumatic diseases. Br $\mathcal{F}$ Rheumatol 1991; 30: $115-8$.

14 Ramirez G, Lambert R, Bloomer H A. Renal pathology in rheumatoid arthritis. Nephron 1981; 2: 124-6.

15 Sellars L, Siamopoulos K, Wilkinson R, Leohapand T, Morley A R. Renal biopsy appearances in rheumatoid arthritis. Clin Nephrol 1983; 20: 114-20.

16 Koota K, Isomamakih H, Mutro O. Death rate and causes of death in RA patients during a period of five years. Scand ₹ Rheumatol 1977; 6: 241-4.

17 Mutru O, Laakso M, Isomäki $H$, Koota K. Ten year mortality and cause of death in patients with rheumatoid arthritis. BMF 1985; 290: 1797-9.

18 Boers M, Dijkmans B A C, Breedveld F C, et al. Subclinical renal dysfunction in rheumatoid arthritis. Arthritis Rheum 1990; 33: 95-101.

19 Bird H A, Yu H, Cooper E H. Renal proximal dysfunction in patients with rheumatic diseases. $B M \mathcal{F} 1984 ; 288$ : 1044-5.

20 Watts G F, Bennett J E, Rowe D J, et al. Assessment of immunochemical methods for determining low concentrations of albumin in urine. Clin Chem 1986; 32: 1544-8.

21 Arnett F C, Edworthy S M, Bloch D A, et al. The American Rheumatism Association 1987 revised criteria for the classification of rheumatoid arthritis. Arthritis Rheum 1988; 31: 315-24.

22 Spencer K, Price C P. Kinetic immunoturbidimetry: the estimation of albumin. Clin Chim Acta 1979; 95: 263-76.

23 Rowe D J F, Dawnay A, Watts G F. Microalbuminuria in diabetes mellitus: review and recommendations for the measurement of albumin in urine. Ann Clin Biochem 1990; 27: 297-312.

24 Duthie J J R, Brown P E, Truelove L H, Baagar F D, Lawrie A J. Course and prognosis in rheumatoid arthritis. A further report. Ann Rheum Dis 1964; 23: 193-204.

25 Boers M. Renal disorders in rheumatoid arthritis. Semin Arthritis Rheum 1990; 20: 57-68.

26 Jacobsson L T H, Knowler W C, Pillemer S, et al. Rheumatoid arthritis and mortality. Arthritis Rheum 1993; 36: 1045-53.

27 Sesso R, Neto J T M, Ferraz M B, Pereira A B, Ajzen H. Frequent abnormalities of renal function in patients with Frequent abnormalities of renal function in pa
rheumatoid arthritis. Nephron 1991; 59: 677-8.

28 Hordon L D, Sellars L, Morley A R, Wilkinson R. Thompson M, Griffiths I D. Haematuria in rheumatoid arthritis: an association with mesangial glomerulonephritis. Ann Rheum Dis 1984; 43: 440-3.

29 Pollet S, Depner T, Moore P, Olander H, Robbins D. Mesangial glomerulopathy and IgM rheumatoid factor in rheumatoid arthritis. Nephron 1989; 51: 107-11.

30 Seideman P, Müller-Suur R. Renal effects of aspirin and low dose methotrexate in rheumatoid arthritis. Ann Rheum Dis 1993; 52: 613-5.

31 Blackshear J L, Napier J S, Davidman M, Stillman M T. Renal complications of non steroidal anti-inflammatory drugs: identification and monitoring of those at risk. drugs: identification and monitoring of
Semin Arthritis Rheum 1985; 14: 163-75.

32 Korpela M, Mustonen J, Pasternack A, Helin H. Mesangial glomerulopathy in rheumatoid arthritis patients. Clinical follow-up and relation to antirheumatic therapy. Nephron 1991; 59: 46-50

33 Gosling P, Shearman C P. Increased levels of urinary proteins: Markers of vascular permeability? Ann Clin Biochem 1988; 25: S150-1.

34 Kushner I. The phenomenon of the acute phase response. Ann NY Acad Sci 1982; 389: 38-48. 